\title{
Spectral Divergence for Cultural Heritage applications
}

\author{
A. Plutino;XLIM laboratory JRU CNRS 7252; Poitiers University; Futuroscope/France \\ N. Richard;XLIM laboratory JRU CNRS 7252; Poitiers University; Futuroscope/France \\ H. Deborah; The Norwegian Colour \& Visual Computing Laboratory, Dpt of Computer Science, NUS\&T; Gjøvik, Norway \\ C. Fernandez-Maloigne;XLIM laboratory JRU CNRS 7252; Poitiers University; Futuroscope/France \\ N. G. Ludwig; Dept. of Physic; State University of Milano; Italy
}

\begin{abstract}
Using reflectance spectra allows to compare the pigment mixtures in paintings. In order to improve the actual subjective spectral comparison, we propose to use spectral similarity measures. The Kullback-Leibler spectral Pseudo-Divergence (KLPD) is selected due to his expected metrological properties. The comparison between a subjective assessment and the objective assessment is developed for mixture of pigments coming from a cultural heritage painting. The obtained results show the good quality of the relationship between the subjective results and the objective ones using the KLPD.
\end{abstract}

\section{Introduction}

Acquiring spectra from artwork is now a current task for cultural heritage preservation or conservation $[7,10,6,17,5,8]$. Nevertheless, comparing spectra in a metrological way is actually a challenge. Even if such results are expected in order to improve the detection of copies, to assess the degradation evolution or to propose solutions for restoration purposes. Actually the main response to this challenge are based on subjective assessment of the spectral variations $[4,2,9]$ or by translating the purpose into the trichromatic domain $[14,23]$, loosing so all the spectral accuracy.

In parallel, recent advances in the metrology of spectral data allowed to produce a first metrologically valid measure of spectral similarity : the Kullback-Leibler spectral Pseudo-Divergence (KLPD)[22]. One of the particularity of this measure is to naturally split the spectral difference into a sum of an intensity and a shape difference. Thanks to this decomposition, the authors proposed to define bi-dimensional histograms of spectral differences (BHSD) in order to analyse the spectral diversity in hyperspectral images[11].

Inside this work, we propose to study the quality of the relationship between a subjective comparison of the spectrum similarities and an objective measure of these similarities using the KLPD. Bi-dimensional representations (objective and subjective) of the spectral differences are used to perform the comparison. In addition, we ask ourselves about the necessity to use this recent measure in confront with the most used ones (Spectral Angle Mapper and Euclidean distances). This work is developed in a real case coming from our cultural heritage.

After having shortly defined the relationship between the spectra and the optical properties of the pigments, we expose the used spectral difference and similarity measures (Euclidean, SAM and KLPD). Then, we develop the used dataset, coming from acquired and expertised data from a real painting and the parameters of the comparison. The comparison results are provide and commented before a short conclusion.

\section{Spectra in Cultural Heritage $(\mathrm{CH})$}

The protection, conservation or restoration require accurate scientific analysis, and if possible using non-destructive testing. In this way, characterizing materials, mixing products and pigments is crucial to study the provenance, the authentication and to improve the datation of artworks. In this way, the spectrometry methods based on the electromagnetic radiation and considered as non-invasive for the support, present efficient solutions.

When an electromagnetic radiation interacts with the matter, the energy is decomposed in absorbed, transmitted and reflected parts. The spectrometry methods registers the correlation between the reflectance of the energy and the wavelength. The reflectance (R) of a surface is usually expressed in percentage of the reflected light $I_{r}$ from the incident one $I_{i}$ :

$$
S=\frac{I_{r}}{I_{i}}
$$

The reflectance measure is fundamental in the study of the materials, because the properties of absorbance, and so the ones of reflectance, are linked to the chemistry of the matter and also to the thickness of the painted layer:

$$
\frac{I_{1}}{I_{0}}=e^{-k_{\lambda} l}
$$

The physical nature of the reflectance spectrum $S$ induces to consider it as a function over the wavelength $\lambda$ defined on a fixed spectral support, $S: s(\lambda), \forall \lambda \subset\left[\lambda_{\min }, \lambda_{\max }\right]$

$$
\begin{aligned}
s: & \mathbb{R} \mapsto \mathbb{R} \\
& \lambda \mapsto r, \text { with } r \subset[0.0,1.0] \text { for reflectance values }
\end{aligned}
$$

The spectral acquisition is performed by digital system using spectral sampling. Nevertheless, in order to be independent from the spectral acquisition sensor and his specific spectral sampling, we will keep the continuous integrals in the following parts of this work.

\section{Spectral Distance and Similarity}

The easiest and the most used way to process the difference between two spectra is to consider them as vectors. In a direct reaction, the most used distance between spectra is a $L_{2}$ Minkowski norm $[15,3,13]$.

$$
d_{L 2}\left(S_{1}, S_{2}\right)=\sqrt{\int_{\lambda_{\min }}^{\lambda_{\max }}\left(s_{1}(\lambda)-s_{2}(\lambda)\right)^{2} d \lambda}
$$


Following this consideration of vectors, some authors searched to be independent from the white mixing, using a vector angle as measure rather than the $L 2$ distance. The Spectral angle Mapper (SAM) measure is then naturally defined as the normalised dot product between two multivariate vectors[16, 21]:

$$
\begin{aligned}
d_{S A M}\left(S_{1}, S_{2}\right) & =\arccos (1-P) \\
P & =\frac{\int_{\lambda_{\min }}^{\lambda_{\max }} s_{1}(\lambda) s_{2}(\lambda) d \lambda}{\int_{\lambda_{\min }}^{\lambda_{\max }} s_{1}(\lambda)^{2} d \lambda \cdot \int_{\lambda_{\min }}^{\lambda_{\max }} s_{2}(\lambda)^{2} d \lambda}
\end{aligned}
$$

In [20], Nidamanuri wondered about the limits of such approaches for spectrum intraclass and interclass discrimination. In particular, the authors developed the fact that SAM measure is more adapted to discriminate dissimilar materials (vegetation from non-vegetation). And in an opposite manner, the Euclidean distance $\left(L_{2}\right.$ norm) was identified as more adapted to measure variations inside spectral groups. Thanks to these considerations, Nidamanuri constructed empirically a similarity score combining the two measures (Normalised Spectral Similarity Score).

Following a parallel way, in [22], the authors proposed a spectral similarity measure combining also a shape $\Delta C$ and an intensity difference $\Delta W$. However, this construction inherits from the Kullback-Leibler divergence measure (known as spectral divergence $[19,18])$. The theoretical construction induced to adapt the Kullback-Leibler divergence using normalised spectra $\bar{S}$ (eq. 8). The obtained Kullback-Leibler spectral pseudo-divergence is then defined by:

$$
\begin{aligned}
& \operatorname{div}_{K L^{\prime}}\left(S, S_{r e f}\right)=\Delta C\left(S, S_{r e f}\right)+\Delta W\left(S, S_{r e f}\right) \\
& \text { with }\left\{\begin{array}{l}
\Delta C\left(S, S_{r e f}\right)=k 1 \cdot K L\left(\bar{S}, \overline{S_{r e f}}\right)+k 2 \cdot K L\left(\overline{S_{r e f}}, \bar{S}\right) \\
\Delta W\left(S, S_{r e f}\right)=\left(k_{1}-k_{2}\right) \log \left(\frac{k_{1}}{k_{2}}\right)
\end{array}\right.
\end{aligned}
$$

considering the normalised spectrum $\bar{S}$ and the total energy $k$ of $S$ as defined by:

$$
\begin{gathered}
\bar{S}=\quad\left\{\bar{s}(\lambda)=\frac{s(\lambda)}{k}, \forall \lambda \in\left[\lambda_{\min }, \lambda_{\max }\right]\right\} \\
\text { with } k=\int_{\lambda_{\min }}^{\lambda_{\max }} s(\lambda) d \lambda .
\end{gathered}
$$

Splitting the spectral difference in two combined measures attached to basic spectral characteristic, present a direct interest in the analysis of spectral set, in particular in the relationship with the human judgement of spectral similarities. Thanks to this construction, we propose to display bi-dimensional representations of the spectral differences, interrogating so the similarity measure ability to discriminate spectral groups.

In $[12,22]$ it was shown that the KLPD is, for the moment, the only one spectral difference measure respecting the metrological constraints, by opposition to the used approaches based on Minkoswki metrics, and associated spectral angles. Nevertheless, following the Nidamanuri construction, we search to identify if such metrological property must be necessary. So, in the following, we propose another kind of bidimensional representation based on the $d_{S A M}$ and $d_{L 2}$ measure. As the $d_{S A M}$ measure assesses the spectral shape variations (dot product), it can be compared to the spectral shape difference $\Delta S$. In the same manner, the $L_{2}$ difference is more sensitive to the intensity change, as the $\Delta W$ measure. This empirical construction induced two theoretical limits. Firstly the two axis are not independent.

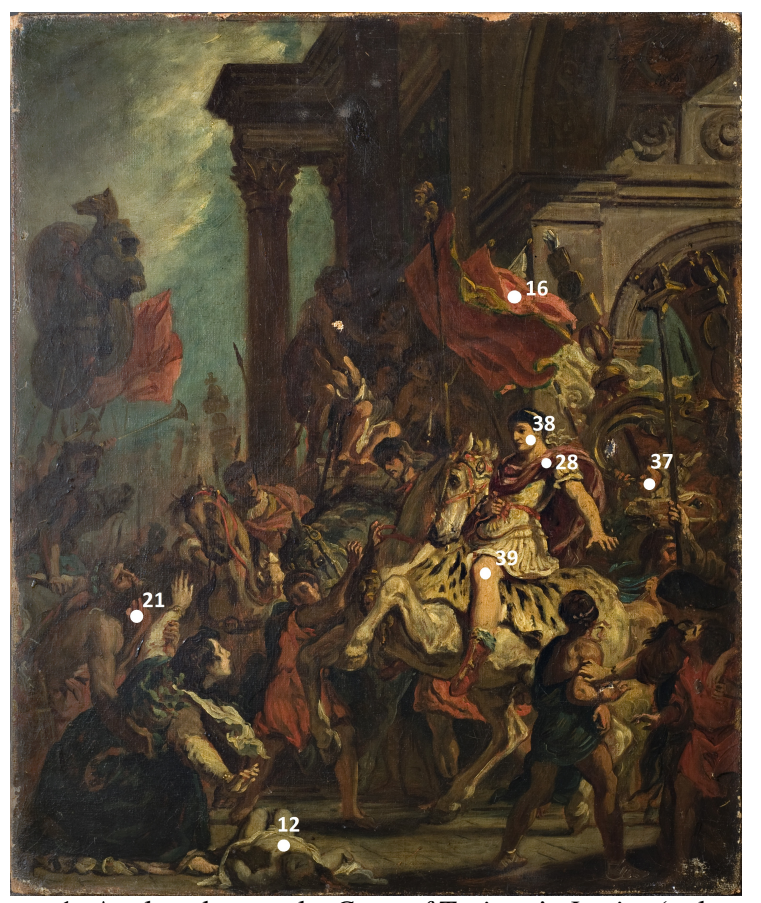

Figure 1: Analysed artwork: Copy of Traiano's Justice (unknown author) and spatial locations of the considered spectra acquired using a FORS system.

\section{A first Experiment The dataset}

The spectral data The used dataset comes from the analysis of a painting (Copy of Traiano's Justice coming from an unknown author) of a private collection. The analyses devoted to elemental and optical characterization of pigments were developed by the DIART Laboratory - diagnostica per l'arte, physics department, University of Milan.

The applied technique is the FORS (Fiber Optic Reflectance Spectroscopy). The data were collected using an Ocean Optics spectrophotometer (HR4000), with spectral sensitivity ranging between 360 and $1100 \mathrm{~nm}$ (spectral resolution of $0,27 \mathrm{~nm}$, see table 1). The selected lightning is a Xenon source (HPX-2000). The lightning/acquisition angle between the surface to acquire, the reflection and backscattering probes is fixed to $45^{\circ}$ thanks to a dedicated support. A Y QR400-7-VIS-NIR fibre model, that avoids specular reflection, was used for the acquisition. The calibration protocol used a white $(R \approx 99 \%)$ and a black $(R \approx 2 \%)$ references. The table 1 resume the used acquisition parameters. In this work only the visible part of the spectra $(380-780 \mathrm{~nm})$ was taken into account.

Table 1: FORS parameters

\begin{tabular}{r|c} 
Integration Time & $100(\mathrm{~ms})$ \\
\hline Spectral Average & $5(\mathrm{~nm})$ \\
\hline Time & $100 \times 5=500(\mathrm{~ms})$ \\
\hline Boxcar & 7
\end{tabular}


Table 2: Identified composition of the colours thanks to the human analysis of the acquired spectra

\begin{tabular}{c|c|c} 
Spectra's Name & Color & Pigment \\
\hline 12.Dead & Rosy & Vermilion and lead white \\
16.Flag & Red & Vermilion \\
21.CapeL & Red & Vermilion \\
28.TCape & Red & Red varnish \\
37.Blower & Rosy & Vermilion and lead white \\
38.TFace & Rosy & Vermilion, red varnish and lead white \\
39.TKnee & Rosy & Red varnish and lead white
\end{tabular}

Subjective pigment analysis Several pigments were analysed, inducing spectral acquisition at different locations in the painting (fig. 1). In this work, we are focusing on a particular red, and more specifically to colours close to the vermilion. The corresponding spectral responses were compared to pigment references (dedicated database from the IFAC [1] ) by two experts. Table 2 presents the proposed pigment nature and mixing when necessary.

In order to improve the pigment characterization/discrimination, an Energy-dispersive X-ray fluorescence spectroscopy $(E D-X R F)$ was performed in order to obtain a chemical characterization of the painting surface, so the pigments. A portable XRF spectrometer (LITHOS 3000, Assing) employing the monochromatic radiation of molybdenum ( $(\mathrm{Mo}, \mathrm{K}=17,4 \mathrm{KeV})$ was used. The $E D-X R F$ detected a great presence of lead $(P b)$ in all the analysed locations. This presence is characteristic from the white lead and from the common preparation layer in paintings. The red points under $E D-X R F$ analysis were 12.Dead, 16.Flag and 38.TFace and the instrument detected the presence of mercury $(\mathrm{Hg})$, characteristic of the vermilion and iron $(\mathrm{Fe})$, typical element for the ochre, but also trace element of many pigments and preparations.

Subjective Bidimensional representation of the spectral similarities Our objective is to compare the experts way to discriminate pigments by spectra with the proposed approach using the Kullback-Leibler spectral pseudo-divergence (KLPD). So, we ask to the expert to organize in a bidimensional representation the spectral differences using the notion of intensity and shape differences. In such construction, the quantitative values have no sense, but the order on each axis are preserved in front of the expert judgement.

The value of intensity correspond to the increase of reflectance associated with the white lead, than, the shape difference value is based on the shape difference between the analysed spectrum and the vermilion reference from the Milano's Laboratory database. The obtained bidimensional representation can be observed in figure $2 b$.

Firstly, we observe that naturally more than one half of the red pigments (red circle) are close from the representation origin (vermilion). Secondly, the closest colours are the pure vermilion (12.Dead and 16.Flag). Then the 3 others are organised in front of the quantity of the added and mixed pigments. Thirdly, a second group of colour appears at the bottom right of the representation (inside the blue circle), including the 28.TCape and 39.TKnee locations. These two colours was identified as formed by red varnish. The observed spectra in figure $2 \mathrm{a}$ shown why the spectral shape and intensity was judged as very different (28.TCape and 39.T Knee are the two lowest spectra). Finally, the

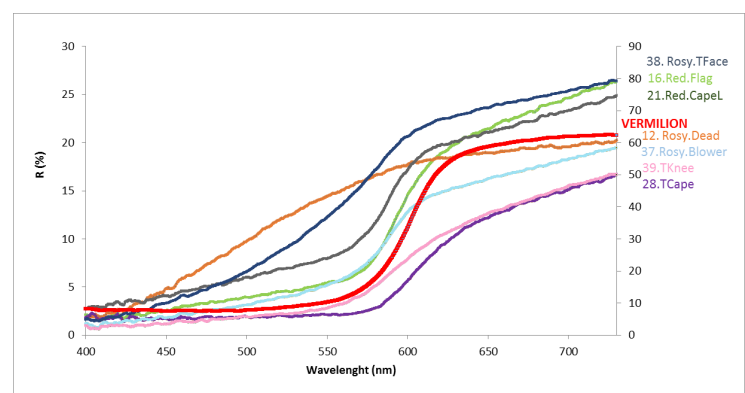

(a) Acquired spectra (vermilion used the dynamic range on the right)

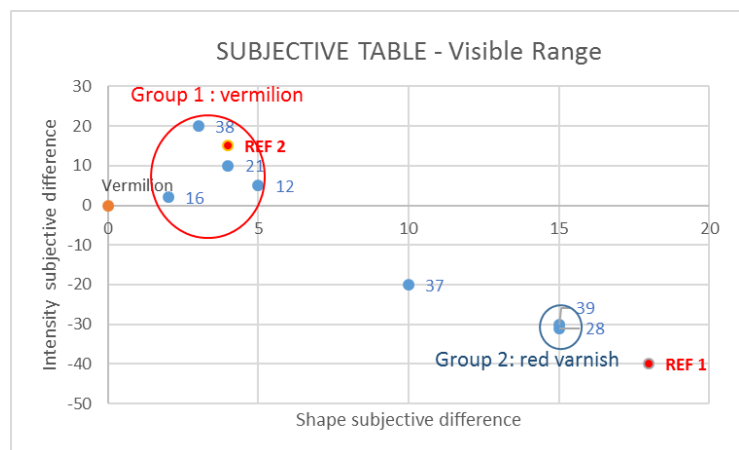

(b) Bidimensional Representation of subjective shape and intensity differences

Figure 2: Acquired spectra from the selected location pointed out in figure 1 and the subjective representation of the spectral differences between the acquired spectra.

colour corresponding to 37. Blower is located between the vermilion and the varnish group. This behaviour of the spectrum could be due to the presence of a third pigment or to a mixture of the first two.

\section{Results}

The references Before to draw the bidimensional representation of spectral differences (BHSD), we need to select the reference to use. As defined previously, the reference must be outside the spectral dataset and stay close from it in order to increase the discrimination ability. In a first consideration, we could imagine to choose the vermilion spectrum as reference. Unfortunately, this spectrum don't respect the second constraint (his intensity level is too important and the considered spectrum is then too far from the spectral data to analyse, see figure 5).

In order to respect the defined constraints, we selected to analyse the spectra using two references framing the spectral set. The reference $\operatorname{Ref} 1$ is selected to be under the spectra with a shape (the step part) slightly sliced to the right of the 28.TCape spectrum. The second reference Ref 2 is by opposition located upper the spectra 38.TFace. Thanks to this direct construction, the constraints are solved and the spectra can be compared using the bidimensional representation of spectral differences.

As previously expressed, we propose two kinds of representation. First ones (fig. 3) are based on a combination of an Euclidean and a spectral angle mapper measures (SAM). The second ones (fig. 4) are based on the two parts of the Kullback-Leibler spectral pseudo-divergence. In the two cases, we mark using blue and red ellipsods, the spectral groups identified using the FORS 


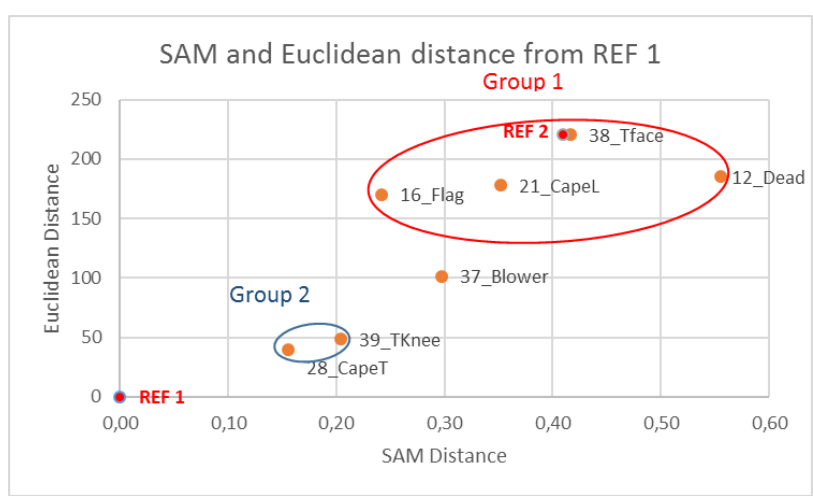

(a) spectral difference to reference 1

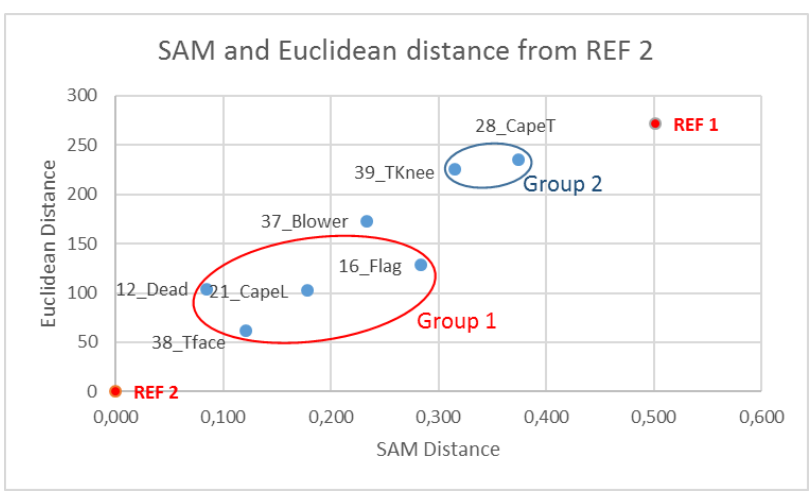

(b) spectral difference to reference 2

Figure 3: Bidimensional representation of the spectral difference using a combination of a Spectral Angle Mapper(SAM) as shape difference and an Euclidean measure as intensity difference.

combined with ED - XRF and the human assessment.

Spectral distribution relative to reference $\operatorname{Ref} 1$ Firstly we consider fig. 3a and 4a. The two spectral representations seems very similar. The two main groups are well separated, and the spectrum 37.Blower is, as expected, located between these two groups. Secondly, the spectra 28.TCape and 39.T Knee are very close from the representation origin (location of the Ref 1 spectrum). Some differences exist between the two representations. Typically, in the relative location of the spectrum 37.Blower to the 16.Flag on the horizontal axis (shape or angle difference). Some inversions can be observed on the vertical axis (intensity difference) : Ref 2 versus 38.T face or 28.TCape versus 39.T Knee.

Spectral distribution relative to $\operatorname{Re} f 2$ Considering the second subset of representations ( $3 \mathrm{~b}$ and $4 \mathrm{~b}$ ), firstly, we note that the 39.TKnee and 28.TCape spectra are located in top-right corner of the representation, farthest of the Ref 2 spectrum. Secondly, we observe that the relative order of the spectra are not preserved from the first representations. Such fact is normal due to the problem dimensionality and to the positive nature of the difference measures. It is interesting to remark also that the intra-class dynamic in the two bidimensional representations are different due to the expressions used to assess the shape and intensity differences. In addition, we remark also the important dynamic of the KLPD representation for the shape difference by opposition to the

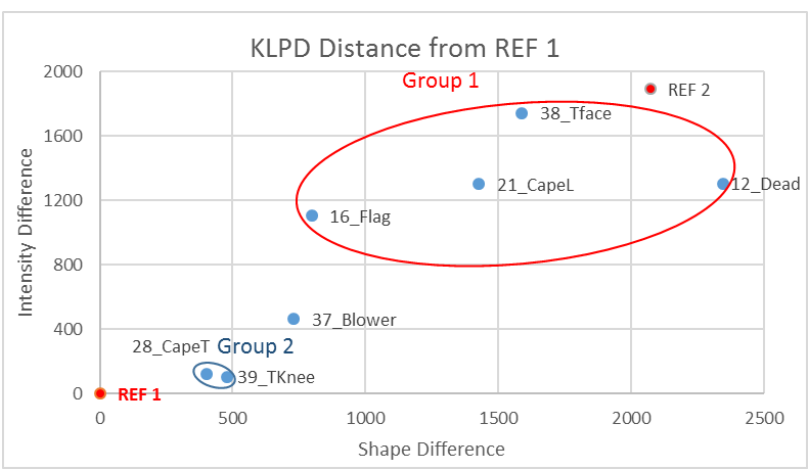

(a) spectral difference to reference 1

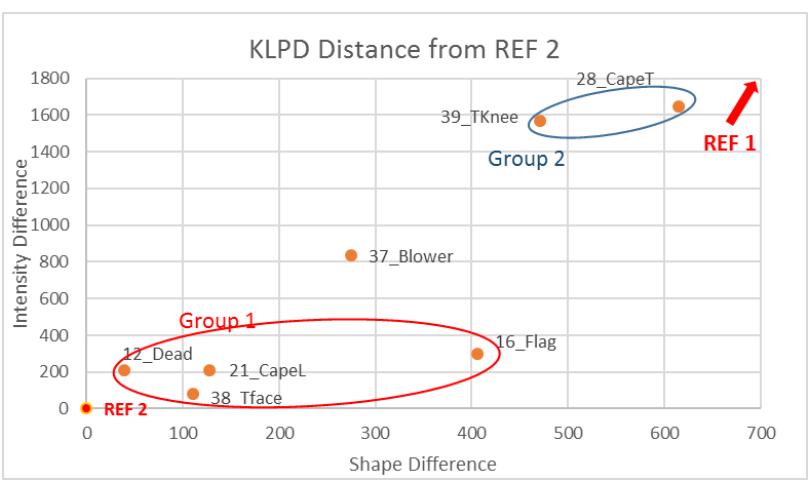

(b) spectral difference to reference 2

Figure 4: Bidimensional representation of the spectral difference using a Kullback-Leibler spectral pseudo-divergence.

SAM measures restricted to $[0,1]$.

\section{Discussion}

Looking at the figures $4 \mathrm{a}$ and $3 \mathrm{~b}$ is possible to discriminate the spectra due to their shape and intensity differences. This is an advantage when analysing pigment's spectra because the shape is strictly correlated with the absorption bands of the materials and the intensity is linked with the lightness and the presence of white in the mixture.

The results obtained through the spectral difference confirms the subjective judgement dividing the pigments in two main groups, but the KLPD gives us more interesting informations about the different spectra inside the groups. In this way, we have the certitude of two main pigments used to make the red colour, but we can also have a clear discrimination of the pigments that presents more white inside them. In fact Group 1 forms a coherent group made with the same pigment, vermilion, but the differences in shape and intensity indicates us the presence of white that increase the intensity and tend to change the spectra's shape. Another interesting point is spectra 37.Blower, because thanks to KLPD we have the confirm that it is a mixture of two different pigment, but we can also know that it is more similar to the Group 1 .

From the results that we obtained, it should be possible in a quick approximation to consider the two kinds of representations as equivalent. Nevertheless the observed differences must be taken with care. If we focus on the easiest measure to analyse, the intensity difference, the expected measure will consider 


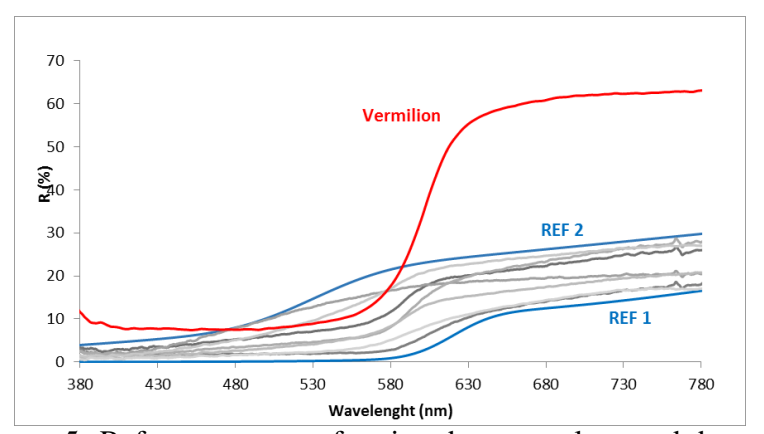

Figure 5: Reference spectra framing the spectral set and the vermilion spectrum .

the relative difference of intensity. This measure is naturally link to the integral of the considered spectra. Having a look on the 21.TCape, 39.T Knee and Ref 1 spectra (figure 2a) we see that the expected order would locate 39.T Knee as being the closest in intensity to the Ref 1 spectrum. Only the representation based on the KLPD measure obtains this result, that is easily confirmed by the integral values. In a more theoretical point of view, we need to not forget that the Euclidean distance embed a part of the spectral angle mapper, inducing so his own limits for the construction of the intensity axis.

So, the main advantage of KLPD measure is then to have a natural decomposition of the spectral difference into intensity and shape differences inherited from the Kullback-Liebler divergence rewriting, instead of an empirical construction. However, this construction of the KLPD allows to compute the total spectral distance in the representation as a $L 1$ norm and consequently to take into account the relative position of two spectra combining the two dimensions. In an opposite manner, the empirical construction of the SAM/Euclidean constructions don't allow such total difference computation due to the dependency of the two axis. This difference is fundamental for spectral analysis, which describes the physical properties of a material.

The main limit of the presented results is link to the analysis of only one pigment from only one painting. As our purpose was to develop and analyse the relationship between theoretical spectral difference measures and our traditional way to compare pigment spectra, the database size is not a problem. We are in front of two new representations, an empirical one improving the Nidamanuri construction [20], and a construction theoretically defined for metrological purposes. One of the questions to solve was about the necessity to choose the KLPD construction, that seemed more complex. The obtained representations show that the results interpretation are direct, and more correlated to our way to consider the spectral similarity. Increasing the number of analysed pigments will not change this result.

The following question, on this way to produce tools for expert in cultural heritage, is linked to the automatic pigment detection in painting (unmixing problem), or to the statistical analysis of the spectral distributions. In such case, the bidimensional representation is transformed into a bidimensional histogram of spectral differences (BHSD)[22]. Such bidimensional histograms are adapted for clustering/classification approaches. Nevertheless, also in this case a total distance of a spectrum to a reference in the bidimensional representation is required, requiring so the KLPD distance rather than the SAM/Euclidean ones.

\section{Conclusion}

Inside this work we explored the interest of recent advances in spectral differences assessment in order to help the experts in cultural heritage for pigment analysis. Even if it exists several spectral distances and classification tools for hyperspectral data, none reach the metrological level before the Kullback-Leibler pseudo-divergence and associated histogram of differences.

One of the interests of the pseudo-divergence is to split the spectral difference into a shape and an intensity differences allowing so the bi-dimensional representation. We proven the performance of the bi-dimensional histogram of differences based on the proposed pseudo-divergence in front of an alternative using the two mainly used spectral distances (Spectral Angle Mapper and Euclidean distance). This result reinforces the limits of nonmetrological approaches for spectral image/data processing.

The result confrontation to the experts judgement imposed to create bi-dimensional representation of his perception of the spectral differences. Creating so a subjective representation for which the ordering of the spectral differences were preserved under his own judgement. The construction was enabled (but not commented) by the a-priori knowledges about the pigment analysis by XRF measures. The obtained comparisons proven the direct relationship between the subjective way to proceed by the expert and the objective and metrological processing obtained by the pseudo-divergence using the histograms of differences.

Thanks to this first results, more complete works are in course in order to address the question of the spectral diversity analysis, or automatic pigment detection from these $n$ dimensional representations of spectral differences. Such results are expected in the scientific tools for cultural heritage analysis, in order to improve the analysis using statistical points of view.

\section{Acknowledgement}

This research work is supported by the DigiPi (Digital Pigment) project funded by the french Research National Agency (ANR) under the number ANR-16-CE08-0010-01, and for parts by the PatAttriMetro project funded by the NUMERIC/ePatrimoine project (ERDF 2016-2020 project) supported by the Aquitaine-Limousin and Poitou-Charentes region. Thank to Letizia Bonizzoni PhD and dott. Marco Gargano of DIART laboratory Milano.

\section{References}

[1] Ifac - cnr database for fors spectra.

[2] M. Aceto, A. Agostino, G. Fenoglio, and M. Picollo. Noninvasive differentiation between natural and synthetic ultramarine blue pigments by means of $250900 \mathrm{~nm}$ fors analysis. Analytical Methods, 5:4184 - 4189, 2013.

[3] C. C. Aggarwal, A. Hinneburg, and D. A. Keim. On the surprising behavior of distance metrics in high dimensional spaces. In Proceedings of the 8th International Conference on Database Theory, ICDT '01, pages 420-434. SpringerVerlag, London, UK, UK, 2001.

[4] A. Atrei, F. Benetti, S. Bracci, D. Magrini, and N. Marchettini. An integrated approach to the study of a reworked painting madonna with child attributed to pietro lorenzetti. Journal of Cultural Heritage, 15:80-84, 2014.

[5] M. Bacci, D. Magrini, M. Picollo, and M. Vervat. A study 
of the blue colors used by telemaco signorini (18351901). Journal of cultural heritage, 10:275 - 280, 2009.

[6] P. Baraldi, S. Bracci, E. Cristoferi, S. Fiorentino, M. Vandini, and E. Venturi. Pigment characterization of drawings and painted layers under 5 th 7 th centuries wall mosaics from ravenna (italy). Journal of cultural heritage, 21:802-808, 2016.

[7] L. Bonizzoni, S. Bruni, A. Galli, M. Gargano, V. Guglielmi, N. N. Ludwig, and M. Martini. Non-invasive in situ analytical techniques working in synergy: the application on graduals held in the certosa di pavia. Microchemical Journal, 126:172-180, 2016.

[8] T. Cavaleri, A. Giovagnoli, and M. Nervo. Pigments and mixtures identification by visible reflectance spectroscopy. Procedia Chemistry, 8:45 - 54, 2013.

[9] E. Cheilakou, M. Troullinos, and M. Koui. Identification of pigments on byzantine wall paintings from crete (14th century ad) using non-invasive fiber optics diffuse reflectance spectroscopy (fors). Journal of Archaeological Science, 41:541 - 555, 2014.

[10] C. Cucci, L. Bigazzi, and M. Picollo. Fibre optic reflectance spectroscopy as a non-invasive tool for investigating plastics degradation in contemporary art collections: Amethodological study on an expanded polystyrene artwork. Journal of Cultural Heritage, 14:290-296, 2013.

[11] H. Deborah. Towards spectral mathematical morphology, 2016.

[12] H. Deborah, N. Richard, and J. Y. Hardeberg. A comprehensive evaluation on spectral distance functions and metrics for hyperspectral image processing. Selected Topics in Applied Earth Observations and Remote Sensing (JSTAR), IEEE Journal of, 8:3224-3234, 2015.

[13] M.-M. Deza and E. Deza. Dictionary of Distances. Elsevier Science, Amsterdam, The Netherlands, 2006.

[14] I. Garofano, J. L. Perez-Rodriguez, D. Robador, and A. Duran. An innovative combination of non-invasive uvvisiblefors, xrd and xrf techniques to study roman wall paintings from seville, spain. Journal of cultural heritage, 22:1028 1039, 2016.

[15] J. C. Gower. A general coefficient of similarity and some of its properties. Biometrics, 27(4):857-871, December 1971.

[16] F. Kruse, A. Lefkoff, J. Boardman, K. Heidebrecht, A. Shapiro, P. Barloon, and A. Goetz. The spectral image processing system (SIPS) - interactive visualization and analysis of imaging spectrometer data. Remote Sensing of Environment, 44(2-3):145-163, 1993.

[17] N. Ludwig and L. Bonizzoni. Misurare l'arte. Vol.1. 2016.

[18] A. Martinez-Uso, F. Pla, J. Sotoca, and P. Garcia-Sevilla. Clustering-based hyperspectral band selection using information measures. Geoscience and Remote Sensing, IEEE Transactions on, 45(12):4158-4171, Dec 2007.

[19] G. Mercier and M. Lennon. On the characterization of hyperspectral texture. In Geoscience and Remote Sensing Symposium, 2002. IGARSS '02. 2002 IEEE International, volume 5, pages 2584-2586 vol.5, 2002.

[20] R. Nidamanuri and B. Zbell. Normalized spectral similarity score $n s^{3}$ as an efficient spectral library searching method for hyperspectral image classification. Selected Topics in Applied Earth Observations and Remote Sensing, IEEE
Journal of, 4(1):226-240, March 2011.

[21] J. C. Price. How unique are spectral signatures? Remote Sensing of Environment, 49(3):181 - 186, 1994.

[22] N. Richard, D. Helbert, C. Olivier, and M. Tamisier. Pseudodivergence and bidimensional histogram of spectral differences for hyperspectral image processing. Journal of Imaging Sciences and Technology (JIST), 60(5):050402.1050402.13, 2016.

[23] A. B. T. Tlaczala. Electronic spectroscopy and trichromaticity colorimetry of some inorganic pigments and their mixtures. Dyes and Pigments, 28:47 - 56, 1995.

\section{Author Biography}

Alice Plutino received her BSc in cultural heritage conservation science at the University of Milan (Italy, 2015). She is currently a researcher at the XLIM laboratory (University of Poitiers) for an Erasmus + Placement project during her Master (master in Conservation science for cultural heritage, University of Milan). Her work focuses on the development of spectral analysis tools for the cultural heritage domain.

Noël Richard received his $P h D$ in signal processing from the University of Poitiers (France, 1993), where he then stayed as an Associate Professor. He coordinates axel of the NUMERIC research program, which is dedicated to digital services involving 19 laboratories, 180 researchers, regional and ERDF funds. He is also the technical chair of the CIE TC8-14 (spatio-chromatic complexity definition and assessment). His research activity is focused on metrological color and spectral image analysis and processing.

Hilda Deborah received her BSc in computer science from the University of Indonesia (2010), her MSc from Erasmus Mundus Color in Informatics and Media Technology (2013), and her PhD in computer science from the University of Poitiers in France and NTNU in Norway (2016). She is currently a researcher at the Norwegian Colour \& Visual Computing Laboratory (Colourlab), NTNU. Her work focuses on the development of spectral mathematical morphology framework and applications in the cultural heritage domain.

Christine Fernandez-Maloigne graduated as a computer engineer (1986) and obtained her PhD degree in Image Processing (1989) from the University of Technology of Compiegne, France. She is currently a professor and vice-rector in charge of international relations at the University of Poitiers, co-director of XLim, and co-animator of the topic "Security" of the Labex. Her research activities focus on colour imaging, colour image and video filtering, segmentation, analysis and indexing, compression, watermarking and quality assessment.

Nicola Ludwig graduated in Physics (1991 Univ. of Milan) and PhD in Science for conservation of cultural heritage (2004 univ of Florence). Assistant Professor of Analytical methods for the studies and conservation of artworks. He is currently working in infrared imaging in the field of non-destructive testing on both biology and Cultural Heritage. The main research topics range from infrared thermography for moisture detection to UV-visiblenearIR spectroscopy applied to pigments identification. 